\title{
Emerging Preclinical and Clinical Applications of Theranostics for Nononcological Disorders
}

\author{
Majid Assadi, MD, FASNC a, Narges Jokar, MSc ${ }^{a}$, Anna Yordanova, MD ${ }^{\text {b,c }}$, \\ Ali Gholamrezanezhad, MD, FEBNM, DABR ${ }^{d}$, Abdullatif Amini, MD ${ }^{e}$, \\ Farhad Abbasi, $\mathrm{MD}^{f}$, Hans-Jürgen Biersack, MD ${ }^{\mathrm{b}}$, Azam Amini, MD ${ }^{g}$, \\ Iraj Nabipour, MD ${ }^{h}$, Hojjat Ahmadzadehfar, MD, MSc ${ }^{\text {b,i, * }}$
}

\section{KEYWORDS}

- Theranostics $\bullet$ Nononcological diseases $\bullet$ Infection $\bullet$ Inflammation $\bullet$ Sarcoidosis

- Rheumatoid diseases

\section{KEY POINTS}

- Along with the considerable results of theranostics in tumoral tissues, there has been a strong push to use this approach for nononcological diseases as well.

- Theranostics approaches may have efficient role in management of rheumatic and cardiovascular diseases, as well as the infections including viral, bacterial and fungal.

- There is an emerging need for randomized trials to specify the factors affecting validity and efficacy of theranostic approaches in nononcological diseases.

\section{INTRODUCTION}

Studies in nuclear medicine have shed light on molecular imaging and therapeutic approaches for oncological and nononcological conditions. Theranostic approaches using the same radiopharmaceuticals for the diagnoses and subsequent therapeutics of malignancies have continuously evolved for site-directed molecular imaging and therapy, especially in oncology. Theranostics, one of the remarkable consequences of the Human Genome Project, has added considerable value to personalized medicine as diagnostic and therapeutic methods are performed exclusively per personal genotypes and phenotypes. Molecular pathways and high-throughput omics platforms are used to recognize and extend small molecular probes for these conditions. Theranostic approach, however, has long been used in clinical practice, namely in

\footnotetext{
a Department of Molecular Imaging and Radionuclide Therapy (MIRT), The Persian Gulf Nuclear Medicine Research Center, Bushehr Medical University Hospital, School of Medicine, Bushehr University of Medical Sciences, Bushehr, Iran; b Department of Nuclear Medicine, University Hospital Bonn, Bonn, Germany; c Department of Radiology, Marienhospital Bonn, Bonn, Germany; d Department of Diagnostic Radiology, Keck School of Medicine, University of Southern California, 1520 San Pablo Street, Suite L1600, Los Angeles, CA 90033, USA; ${ }^{\text {e }}$ Bushehr Heart Medical Center, School of Medicine, Bushehr University of Medical Sciences, Bushehr, Iran; ${ }^{f}$ Department of Infectious Diseases, Bushehr Medical University Hospital, School of Medicine, Bushehr University of Medical Sciences, Bushehr, Iran; ${ }^{9}$ Department of Internal Medicine, Division of Rheumatology, Bushehr Medical University Hospital, School of Medicine, Bushehr University of Medical Sciences, Bushehr, Iran; ${ }^{\mathrm{h}}$ The Persian Gulf Tropical Medicine Research Center, The Persian Gulf Biomedical Sciences Research Institute, Bushehr University of Medical Sciences, Bushehr, Iran; ' Department of Nuclear Medicine, Klinikum Westfalen, Am Knappschaftskrankenhaus 1, Dortmund 44309, Germany

* Corresponding author. Department of Nuclear Medicine, Klinikum Westfalen, Am Knappschaftskrankenhaus 1, Dortmund 44309, Germany.

E-mail addresses: hojjat.ahmadzadehfar@ruhr-uni-bochum.de; nuclearmedicine@gmail.com
} 